\title{
Crecimiento económico de pequeñas empresas agrícolas del desierto de Arica: una aproximación a la asistencia e impacto del Centro de Negocios de Sercotec. Arica, Chile
}

\author{
Economic growth of small agricultural businesses in the Arica desert: an approach \\ to the assistance and impact of the Sercotec Business Center. Arica, Chile
}

\author{
Rodrigo Barra Novoa ${ }^{1}$
}

\begin{abstract}
RESUMEN
Este artículo se realizó en el marco del programa de Centros de Desarrollo de Negocios en Chile, orientado a obtener un mayor impacto en el crecimiento y productividad de las empresas de menor tamaño, mediante un conjunto de acciones de apoyo. La iniciativa gubernamental es liderada desde el año 2015 por el Servicio de Cooperación Técnica (SERCOTEC), que inició la implementación de una red de 51 Centros de Desarrollo de Negocios con despliegue nacional, cuyo propósito principal es que "emprendedores, micro y pequeñas empresas de todos los sectores productivos mejoren sus capacidades de gestión y su desempeño económico". La conclusión principal del artículo es que los primeros resultados de impacto económico muestran que las empresas asistidas en la Región de Arica y Parinacota, especialmente del segmento agrícola, exhiben ciertas analogías en la mayoría de las áreas de asesoría empresarial que explican su positivo desempeño en aumento en ventas. También se argumenta que la sostenida capacidad de identificar nuevas oportunidades de mercado y hacer un uso eficiente de sus recursos productivos - por las condiciones de suelo y clima local en pleno desierto de Atacama- las induce a innovar permanentemente, estimulando transversalmente la inserción de sus productos en mercados más rentables, lo cual explica igualmente su mejor desempeño económico.
\end{abstract}

Palabras clave: pequeñas empresas, políticas públicas, crecimiento económico, empresas agrícolas del desierto.

\begin{abstract}
This article developed in the framework of the Business Development Centers in Chile program aims at obtaining a higher impact on the growth and productivity of smaller companies. The initiative led by the Technical Cooperation Service (SERCOTEC) began the implementation of a network of 51 Business Development Centers with national deployment, whose purpose is that "entrepreneurs, micro and small enterprises of all productive sectors improve their management skills and economic performance. The article's main conclusion suggests that the first economic impact results show that the assisted enterprises in the Region of Arica and Parinacota, especially in the agricultural segment, exhibit certain analogies in most areas of business assistance that explain their positive performance in increasing sales. Also, it is argued that the sustained capacity to identify new market opportunities and make efficient use of their productive resources (due to the local soil and climate conditions in the middle of the Atacama Desert) induce them to innovate permanently, stimulating the insertion of their products transversally in more profitable markets, which also explains their better economic performance.
\end{abstract}

Keywords: small enterprises, public policies, economic growth, agricultural companies in the desert.

\section{Introducción}

Las micro, pequeñas y medianas empresas son actores fundamentales en el desarrollo económico y social de Chile, debido a que aportan el 53\% de los empleos y representan el 98,7\% del total de las empresas formales del país. La producción del segmento Mipyme está principalmente vinculada al mercado interno, por lo que una parte representativa de la población y economía nacional depende de su actividad empresarial.

El diagnóstico nacional demuestra que las micro y pequeñas empresas presentan diversas limitaciones que afectan su desempeño industrial.

\footnotetext{
1 Centro de Negocios Sercotec Arica, programa financiado por el Servicio de Cooperación Técnica (SERCOTEC) y operado por la Universidad de Tarapacá (UTA).

* Autor por correspondencia: rodrigo.barra@centrossercotec.cl
}

Fecha de Recepción: 20 de Octubre, 2020.

Fecha de Aceptación: 3 de Enero, 2021. 
Estas barreras retraen a los emprendedores a la hora de embarcarse en la creación de nuevos negocios o provocan que las empresas establecidas operen con baja eficiencia productiva.

Ahora bien, numerosas investigaciones enfatizan una relación positiva entre una buena gestión administrativa y un buen desempeño económico en las empresas. Bloom y Van Reenen (2007) sugieren, a partir de diversos acercamientos científicos y estudios de caso, que mejores prácticas de gestión están fuertemente asociadas con la productividad, la rentabilidad, el crecimiento de las ventas y las tasas de supervivencia de las empresas.

Desde una perspectiva gubernamental, entregar a las micro y pequeñas empresas herramientas para mejorar la gestión, innovación y competitividad, ha constituido un objetivo de política pública en las últimas tres décadas. Existen agencias especializadas en esta tarea como SERCOTEC, institución dedicada desde hace 65 años al apoyo y fortalecimiento de los pequeños negocios del país.

Este esquema institucional impulsa al Gobierno de Chile para avanzar hacia una segunda fase de servicios de apoyo público, orientada a obtener un mayor impacto en el crecimiento y productividad de las pequeñas empresas. Con ese fin, y de acuerdo a lo dispuesto en la Agenda de Productividad, Innovación y Crecimiento (2014-2018), en el 2015 se inició la implementación de la red de Centros de Desarrollo de Negocios con despliegue nacional, cuyo objetivo es apoyar a través de asistencia técnica gratuita y de largo plazo a las Mipymes establecidas y emprendimientos del país (Sercotec, 2018).

La iniciativa impulsada por el Ministerio de Economía, Fomento y Turismo es ejecutada desde sus orígenes por SERCOTEC y se basa en el modelo Small Business Development Centers (SBDC) de los Estados Unidos (SBDC Global, 2019).

Tras la primera evaluación de impacto del programa, aplicada a los primeros 27 Centros de Desarrollo de Negocios, se confirma que la asesoría suministrada tiene un efecto positivo, y económicamente importante, en términos de creación y formalización de empleos, incremento en las ventas y apalancamiento de recursos de inversión. La investigación, además, evidenció a nivel cualitativo que los centros no solo ayudan a crear más puestos de trabajo formal, sino que también inciden en aspectos que contribuyen a mejorar la calidad de la relación laboral entre empresarios y trabajadores.

Concluido el estudio, surge como oportunidad para el programa, evaluar y medir sistemáticamente el impacto de las intervenciones de cada operador.

En esta investigación se analizan los principales resultados de impacto económico de una muestra de empresas y nuevos emprendimientos que recibieron asistencia técnica en el Centro de Desarrollo de Negocios de Arica. Para comprobar empíricamente los impactos obtenidos, se examinó la ficha histórica de 833 clientes asistidos entre los años 2015-2019. A partir de la información disponible en el software Neoserra, se elaboró una base de datos de carácter longitudinal, que da cuenta del incremento en ventas, generación de empleo y financiamiento obtenido, examinando transversalmente la eficiencia del programa, con variables de género, etnia, tamaño y sector económico.

El documento ha sido organizado en seis secciones. La primera sección presenta la metodología y base de datos utilizada; la segunda sección examina la revisión de la literatura; la tercera sección proporciona una breve descripción del programa de Centros de Desarrollo de Negocios en Chile; la cuarta sección evalúa los impactos empresariales obtenidos por los clientes asistidos en el Centro de Arica, especialmente del segmento agrícola; la quinta sección analiza la relación costo-beneficio del programa, y la última sección presenta las principales conclusiones del estudio.

\section{Metodología y base de datos}

La metodología empleada combina técnicas cualitativas y cuantitativas. En primer lugar, la recolección de la información incluyó la revisión de publicaciones, informes técnicos, presupuestos oficiales, manuales de operación y planes de trabajo.

Para comprobar empíricamente los impactos alcanzados por el programa en la Región de Arica y Parinacota, se utilizó el software Neoserra, el cual permitió examinar la historia de 833 clientes asistidos entre los años 2015-2019. A partir de la información disponible, se elaboró una base de datos de carácter longitudinal, que representa la dinámica y crecimiento de los clientes atendidos, y evalúa la relación entre los principales indicadores de desempeño del programa, con otras variables que la literatura sugiere para su tratamiento, entre 
ellas, eficiencia por género, etnia, tamaño y sector económico.

Del total de empresas analizadas, se examinaron con mayor profundidad 383 encuestas de impacto económico.

El incremento de las ventas se sustentó en la revisión de carpetas tributarias (Formulario 29 o declaración mensual de IVA), descargadas de la plataforma del Servicio de Impuestos Internos y registradas en las respectivas encuestas de impacto económico.

La creación de empleos se respaldó a través de la revisión de los contratos de trabajo generados en cada período y la obtención de financiamiento descansó en la revisión de los convenios formalizados entre los clientes asistidos y las instituciones de financiamiento público y privado.

Para obtener información adicional sobre los impactos en el crecimiento de las firmas asistidas, se realizaron tres estudios de caso que permitieron identificar aspectos cualitativos no registrados en la información disponible. Una buena parte de este esfuerzo se enfocó en aquellos clientes que presentaron tasas de aumento en ventas superiores al promedio. En tal sentido, se observa que las firmas, especialmente del segmento agrícola, mejoran su desempeño empresarial a partir de cambios en los modelos de negocios, uso de nuevas tecnologías, apalancamiento de subsidios y acceso a nuevos mercados. Esta lectura apreciativa y estructural del proceso de crecimiento permite reafirmar las conclusiones generales del estudio.

\section{Crecimiento económico basado en los recursos y las capacidades}

Existe una gran discrepancia académica entre los modelos más formales sobre el crecimiento desarrollados por los economistas neoclásicos y las miradas estructuralistas o evolucionistas apoyadas en una visión histórica de los procesos de crecimiento (Nelson, 1982). En los manuales de economía neoclásica se observa que las empresas suelen ser presentadas como una suerte de "caja negra", que de algún modo combinan cierta cantidad de trabajo con cierta cantidad de capital, siguiendo lo postulado por un modelo dominado por la teoría convencional del equilibrio estático.

Indudablemente, la economía neoclásica nos brinda un punto de partida para entender una serie de factores que afectan el crecimiento económico de las empresas. Sin embargo, y dado que estamos en una etapa de rápida transformación tecnológica, en la cual existen diversas posibilidades para estudiar las trayectorias, rutinas y capacidades que afectan la demografía empresarial, nos resulta algo limitado ajustarnos a una modelización estática de los fenómenos del crecimiento.

Edith Penrose en su libro "La teoría del crecimiento de la firma" estudió las causas del crecimiento empresarial y los factores que llevan a limitar su tasa de crecimiento. Al explorar la relación entre los recursos de la empresa y su desarrollo, la autora observó que las firmas son una organización administrativa conformada por un conjunto de recursos, donde el crecimiento está determinado por los recursos que posee y también por una lógica o cultura empresarial de organización y comportamiento (Penrose, 1959).

Para ilustrar este punto, Penrose señaló que las empresas que están dotadas de ciertos recursos, gestionados por "gerentes emprendedores", proveen tanto un incentivo para la expansión como un límite a la tasa de crecimiento. Desde esta perspectiva, la tasa de crecimiento de una empresa está limitada por el conocimiento, la eficacia administrativa y la colección de recursos productivos de que dispone. Estos recursos son en sí mismos un conjunto de servicios potenciales que pueden ser utilizados con mayor o menor eficiencia. Por lo tanto, el tamaño de la empresa es el valor actual total de sus recursos utilizados para fines productivos y la eficiencia con que los emplea. En este último sentido cuenta lo que llamaríamos la cultura empresarial en el uso de los recursos.

Penrose consideraba que una empresa es una ecuación compuesta por la organización administrativa y la recaudación de los recursos productivos. Por tal motivo, las actividades productivas de la empresa se rigen por las oportunidades identificadas por el empresario, y su búsqueda es una disposición emprendedora que requiere de intuición y creatividad.

La autora también argumenta que los límites del crecimiento de la empresa están fijados por la demanda percibida por el empresario y, a medida que aumenta de tamaño, su composición "producto-mercado" cambia en consecuencia. A la vez, la disponibilidad de servicios productivos no utilizados se convierte en un reto para innovar, un incentivo para la expansión y una fuente de ventaja competitiva. 


\section{Crecimiento empresarial desde una perspectiva inductiva y evolutiva}

Gran parte de las teorías que buscan determinar las fuentes de crecimiento empresarial se han sustentado en métodos inductivos y estudios de caso. Sin embargo, durante las últimas décadas muchos investigadores se han alejado de los métodos narrativos para acercarse a técnicas econométricas y estadísticas, utilizando modelos estructurales que permitieran incorporar causalidad a las variables. La motivación básica detrás de esta búsqueda fue la necesidad científica de evaluar la validez estadística de las teorías desarrolladas a partir de la observación casuística de la realidad. En tal sentido, Tarziján señala que, a pesar del atractivo de este impulso hacia una validación estadística de las diversas teorías, no hay consenso en torno al real avance en este frente (Tarziján, J. 2009).

En cuanto a las variables empresariales, se han identificado ciertos factores que se pueden relacionar con una alta rentabilidad y crecimiento (como el tipo de industria, la inversión sistemática en innovación y el cambio tecnológico), pero aún se está lejos de encontrar causalidades claras o de establecer variables específicas que afecten dicha rentabilidad (Tarziján, J. 2009).

Otros autores como Bloom y Van Reenen (2010), tras realizar un amplio programa de investigación para medir sistemáticamente las prácticas de gestión en países desarrollados y en desarrollo, explicaron que las grandes diferencias de productividad entre las empresas y los países -diferencias que no pueden explicarse fácilmente por otros factoresson las variaciones en las prácticas de gestión. En tal sentido, las conclusiones de sus investigaciones señalan que "las empresas con mejores prácticas de gestión tienden a tener un mejor desempeño en una amplia gama de dimensiones: son más grandes, más productivas, crecen en términos de tamaño y productividad, así como en términos de supervivencia”.

Desde una perspectiva evolutiva, Richard Nelson señala que las investigaciones sobre las diferencias entre empresas y las implicaciones en la tasa de supervivencia y crecimiento empresarial han puesto un excesivo énfasis en la modelización formal y análisis cuantitativo, dejando de lado una mejor comprensión de lo que efectivamente está ocurriendo al interior de la firma (Nelson y Winter).
Por ello, Nelson sostiene que la heterogeneidad industrial nos lleva necesariamente a una amplia tipología de empresas y circunstancias, a diferentes grupos de clientes y a una diversidad de patrones de comportamiento.

Si bien es probable que haya solapamiento de mercados, diferentes firmas están ubicadas en diferentes lugares, y tienen una cierta reputación y trayectoria. Algunas de estas empresas están bajo una nueva administración, o han sido recientemente creadas. Otras difieren en las ganancias que obtienen y en la productividad total de los factores (PTF).

Mientras algunas empresas operan con baja productividad y tienen escaso capital de respaldo para alcanzar su punto de equilibrio, otras no muestran interés en expandir sus operaciones, simplemente porque son de propiedad familiar y no ven incentivos para ello (Nelson, 1982).

Otra idea fundamental de Nelson es que, en un entorno industrial, algunas empresas pueden tener altos beneficios vendiendo en un mercado particular, donde la demanda es relativamente inelástica y la expansión de las actividades no necesariamente será tan rentable en el futuro en caso de diversificar a otras áreas de trabajo. A la vez, la variedad y turbulencia de una industria en cualquier momento estarán relacionadas con la importancia relativa de nuevos participantes y sus características.

\section{Centros de Desarrollo de Negocios de Sercotec}

Las políticas de fomento y promoción para las micro y pequeñas empresas se han desarrollado principalmente sobre la base de una institucionalidad pública dependiente de diversos ministerios y agencias especializadas. En este sentido, la contribución del Servicio de Cooperación Técnica (SERCOTEC) ha sido fundamental desde 1952. La corporación de derecho privado, dependiente del Ministerio de Economía, Fomento y Turismo, nació gracias a un acuerdo cooperativo de asistencia técnica al que concurrieron la Corporación de Fomento de la Producción (CORFO) y el Instituto de Asuntos Interamericanos de Estados Unidos (Sercotec, 2018).

Desde la década de los noventa, la institución cuenta con presupuesto regular para apoyar a las pequeñas empresas, destacándose actualmente subsidios y programas como Capital Semilla, Capital Abeja, Crece Multisectorial, Mejora 
Negocios y otras iniciativas de interés público como el programa de Fortalecimiento Gremial y Cooperativo, Barrios Comerciales y Formación Empresarial.

En el año 2015 se puso en marcha el programa de Centros de Desarrollo de Negocios, iniciativa que nació con el acuerdo suscrito entre los Gobiernos de Chile y Estados Unidos en junio de 2014, en materia de promoción del emprendimiento y el crecimiento de las pequeñas empresas. Este acuerdo comprometió asesoría técnica, capacitación y colaboración de los Estados Unidos para la implementación de los centros Chile, inspirados en el modelo Small Business Development Centers (SBDC).

Tras quedar encargado de implementar esta iniciativa, Sercotec modificó su estructura organizacional, y creó la Gerencia de Centros de Desarrollo de Negocios en el nivel central y con alcance regional a través de la designación de un ejecutivo de fomento en cada Dirección Regional.

Es importante mencionar que en los últimos 15 años la institución ya contaba con un presupuesto estable para ejecutar sus programas, pero sin mayor crecimiento. Los centros cambiaron esta situación, con un incremento de los recursos destinados a programas que en el año 2017 alcanzó un 32\%. Esta evolución fue la máxima expresión del cambio de paradigma impulsado por la administración en los distintos programas, que ayudó a pasar del apoyo a la inversión prestado por la institución a la asesoría empresarial, privilegiando el impacto por sobre el número de empresas atendidas.

\section{Modelo de Intervención del Programa}

Los Centros de Desarrollo de Negocios son espacios donde las empresas de menor tamaño y los emprendedores reciben asesoría técnica, individual y sin costo a través de asesores expertos, para fortalecer las capacidades empresariales a largo plazo y asegurar efectivos resultados de impacto económico. Los centros absorben el aprendizaje de espacios de apoyo y orientación a las empresas que en periodos anteriores había dispuesto SERCOTEC, al igual que otras instituciones, como la agencia de desarrollo económico chilena CORFO, con recursos del gobierno central y de gobiernos regionales. La implementación del programa se realizó de manera incremental desde el segundo semestre de 2015, considerando inicialmente la migración de los centros Chile Emprende de CORFO y la incorporación de nuevos centros aprobados por concurso público.

Los centros tienen como foco de intervención la creación de impacto económico en las empresas atendidas, primordialmente a través del aumento de sus ventas y del empleo, midiendo estos resultados empresariales en el tiempo. Para ello incentivan un modelo de trabajo concordado con el emprendedor o empresario, donde este compromete su esfuerzo y constancia en la ejecución de las acciones recomendadas en el marco de la asesoría.

La misión de los centros es promover el crecimiento, la productividad y la innovación de las empresas de menor tamaño, acompañándolas en sus desafíos de desarrollo por medio de asesoría especializada para mejorar su gestión. Los centros aspiran a ser reconocidos como un referente de asesoría técnica y de gestión de alto valor para dichas empresas, contribuyendo a la viabilidad y sostenibilidad de estas y de sus trabajadores.

Las pequeñas empresas y emprendedores reciben asistencia integral según las necesidades de cada negocio. Estas necesidades particulares son identificadas en un diagnóstico inicial que el asesor hace del negocio y del empresario o empresaria. A partir de este diagnóstico se elabora un plan de trabajo individual acordado entre el centro y el asesorado.

El cliente asistido accede a una oferta de servicios de asesoría y capacitación de alto valor a la medida de sus requerimientos, con énfasis en el acompañamiento de largo plazo para asegurar efectivos resultados, que se van midiendo de manera sistemática.

Complementariamente, los centros facilitan el acceso a servicios financieros, investigación de mercado y vinculación con el ecosistema productivo local. Para el último punto, la exigencia a los operadores incluye postulaciones en consorcio con entidades de educación superior, asociaciones empresariales, municipalidades y gobiernos locales. A este respecto, al finalizar el año 2018 el programa contaba con más de 580 socios estratégicos a nivel nacional.

Las acciones de apoyo radican en entregar asistencia técnica especializada, acompañamiento personalizado y seguimiento en sus empresas, y cuando corresponda, capacitación destinada a mejorar las capacidades de gestión empresarial. El segmento de atención de los centros son las 
empresas de menor tamaño, idealmente de los segmentos micro 3 , pequeña 1 , pequeña 2 y pequeña 3 , con ventas que van desde las 600,01 UF hasta las 25.000 UF. También se consideran los emprendedores sin iniciación de actividades.

Los tres niveles de atención son los siguientes:

- Nivel 1: emprendedores/as que desean comenzar un nuevo negocio o están en las etapas iniciales de puesta en marcha de una iniciativa, sin iniciación de actividades ante el Servicio de Impuestos Internos.

- Nivel 2: empresas establecidas que tienen un negocio en operación y buscan oportunidades $o$ formas de mejorarlo o hacerlo crecer, presentan un problema específico o una necesidad que debe ser resuelta y/o están atravesando por una crisis de mercado.

- Nivel 3: empresas gacelas con niveles de crecimiento elevados en comparación con la media de su sector o sus competidores, necesitan asistencia para gestionar adecuadamente su crecimiento y destrabar diversos "cuellos de botella" (Eurostat - OCDE, 2007).

\section{Impacto económico y sectores atendidos por el programa en Arica y Parinacota}

A continuación, se presenta un conjunto de indicadores de impacto y crecimiento empresarial, que más adelante se interpretarán en un marco teórico evolutivo, explicando las transformaciones estructurales acumuladas a lo largo del período analizado.

Los resultados tempranos alcanzados sugieren que la formación y asesoría suministrada por el programa, al ser instructiva y orientada a la aplicación de nuevos conocimientos y capacidades, ayuda a disminuir brechas comunes de conocimiento. También se observa la adopción de nuevos enfoques comerciales que ayudan a mejorar los desempeños y rendimientos de las firmas asistidas. Estos procesos de transformación se sustentan en la relación confidencial, acompañamiento personalizado y seguimiento sistemático de las acciones comprometidas en cada uno de los planes de asistencia empresarial. En la Tabla 1 se identifican los principales indicadores de impacto económico alcanzados al 31 de marzo de 2019.

Tabla 1. Impacto económico obtenido por el Centro de Arica (2015-2019).

\begin{tabular}{|c|c|c|c|c|c|}
\hline \multirow{2}{*}{ Indicador } & Año 1 & Año 2 & Año 3 & Año 4 & \\
\hline & 2015-2016 & 2016-2017 & 2017-2018 & 2018-2019 & $\begin{array}{l}\text { Acumulado/ } \\
\text { Promedio }\end{array}$ \\
\hline Clientes asistidos & 321 & 497 & 457 & 302 & 1.577 \\
\hline Clientes asistidos por asesor & 80 & 107 & 107 & 107 & 100 \\
\hline Horas de asesoría por cliente & 10,3 & 8,7 & 8,0 & 6,0 & 8,25 \\
\hline Empresas que declaran aumento en ventas & 35 & 135 & 140 & 66 & 376 \\
\hline Aumento en ventas (Miles \$) & 205.445 .320 & 1.050 .391 .874 & 1.552 .453 .210 & 1.271 .362 .396 & 4.079 .652 .800 \\
\hline$\%$ de empresas atendidas que aumentan ventas & $10,9 \%$ & $27,5 \%$ & $31,4 \%$ & $21,8 \%$ & $22,9 \%$ \\
\hline \% participación del aumento en ventas generado por Gacelas (N3) & $55,9 \%$ & $41,7 \%$ & $32,5 \%$ & $42,7 \%$ & $39,2 \%$ \\
\hline$\%$ aumento en ventas generado por mujeres & $42,9 \%$ & $57,7 \%$ & $51,7 \%$ & $58,5 \%$ & $52,7 \%$ \\
\hline$\%$ aumento en ventas generado por hombres & $48,6 \%$ & $37,2 \%$ & $43,4 \%$ & $41,5 \%$ & $42,7 \%$ \\
\hline Empleos creados & 37 & 34 & 45 & 46 & 162 \\
\hline Emprendimientos formalizados en primera categoría & 32 & 44 & 41 & 15 & 132 \\
\hline$\%$ emprendimientos formalizados sobre el total de clientes N1 & $36 \%$ & $35 \%$ & $39 \%$ & $41 \%$ & $37 \%$ \\
\hline $\begin{array}{l}\text { Financiamiento obtenido (Ahorro del empresario y créditos } \\
\text { solicitados con apoyo del programa) }\end{array}$ & 183.186 .098 & 121.404 .820 & 171.569 .425 & 213.268 .614 & 689.428 .957 \\
\hline Financiamiento obtenido (Subsidios) & 44.626 .103 & 73.400 .529 & 67.579 .028 & 28.400 .000 & 214.005 .660 \\
\hline
\end{tabular}

Fuente: Elaboración propia a partir de Neoserra. 
La estadística descriptiva resultante de las variables de corte longitudinal, examinadas en las Tablas 2, 3 y 4, muestran las principales diferencias empresariales existentes en función del género, tamaño, sector económico y origen étnico. En tal sentido, aunque las trayectorias y experiencias empresariales son muy heterogéneas, las principales tendencias indican que las empresas lideradas por mujeres y empresas gacelas -las más innovativas- han tenido un mejor desempeño en la tasa de aumento en ventas. A pesar de que las microempresas y el sector de servicios representan una mayor participación en el mismo
Tabla 2. Porcentaje global de aumento ventas por sector económico (2015-2019).

\begin{tabular}{lc}
\hline Sector & $\begin{array}{c}\text { \% de Aumento } \\
\text { en ventas }\end{array}$ \\
\hline Servicios & 54,0 \\
\hline Agricultura & 80,3 \\
\hline Comercio al por mayor y menor & 49,2 \\
\hline Servicio de hospedaje, alimentación y turismo & 64,6 \\
\hline Transporte y almacenamiento & 13,0 \\
\hline Manufactura & 42,5 \\
\hline Otros sectores & 50,6 \\
\hline
\end{tabular}

Fuente: Elaboración propia a partir de Neoserra.

Tabla 3. Principales requerimientos de asesoría por sector económico (2015-2019).

\begin{tabular}{lccccc}
\hline Sector & $\begin{array}{c}\text { Administración } \\
\text { de empresas }\end{array}$ & $\begin{array}{c}\text { Plan de } \\
\text { Negocios }\end{array}$ & $\begin{array}{c}\text { Mercadotecnia } \\
\text { y ventas }\end{array}$ & $\begin{array}{c}\text { Financiamiento } \\
\text { Apertura de } \\
\text { negocios }\end{array}$ \\
\hline Servicios & 26,3 & 27,5 & 20,3 & 13,4 & 12,6 \\
\hline Agricultura & 27,1 & 26,8 & 24,4 & 15,0 & 6,7 \\
\hline Comercio al por mayor y menor & 26,3 & 19,2 & 30,2 & 15,1 & 9,15 \\
\hline Servicio de hospedaje, alimentación y turismo & 25,8 & 33,8 & 15,1 & 17,4 & 7,8 \\
\hline Transporte y almacenamiento & 21,3 & 27,6 & 17,6 & 26,2 & 7,2 \\
\hline Manufactura & 28,7 & 23,8 & 22,7 & 13,8 & 11,0 \\
\hline Otros sectores & 27,7 & 26,1 & 19,0 & 15,0 & 12,1 \\
\hline
\end{tabular}

Fuente: Elaboración propia a partir de Neoserra.

Tabla 4. Participación del aumento en ventas por tamaño, sector económico y origen étnico.

\begin{tabular}{|c|c|c|c|c|c|}
\hline & Año 1 & Año 2 & Año 3 & Año 4 & \\
\hline & $2015-2016$ & $2016-2017$ & $2017-2018$ & $2018-2019$ & Promedo \\
\hline Micro & 28,6 & 61,4 & 61,9 & 63,5 & 53,9 \\
\hline Pequeña & 55,2 & 34,4 & 32,6 & 35,6 & 39,4 \\
\hline Mediana & 16,2 & 4,3 & 5,4 & 0,9 & 6,7 \\
\hline Servicios & 20,6 & 29,6 & 38,9 & 59,4 & 37,1 \\
\hline Agricultura & 4,2 & 10,3 & 4,7 & 14,4 & 8,4 \\
\hline Comercio al por mayor y menor & 29,5 & 34,2 & 23,9 & 7,9 & 23,9 \\
\hline Servicios de hospedaje, alimentación y turismo & 9,1 & 7,9 & 5,9 & 11,2 & 8,5 \\
\hline Transporte y almacenamiento & 17,1 & 2,9 & 5,6 & 0 & 6,4 \\
\hline Manufactura & 1,0 & 8,2 & 3,5 & 6,4 & 4,8 \\
\hline Otros sectores & 18,6 & 6,9 & 17,4 & 0,8 & 10,9 \\
\hline Con etnia (aymara, quechua, otro) & 28,5 & 33,3 & 30,7 & 34,8 & 31,8 \\
\hline Sin etnia (No declara pertenecer a etnia originaria) & 71,5 & 66,7 & 69,3 & 65,2 & 68,2 \\
\hline
\end{tabular}

Fuente: Elaboración propia a partir de Neoserra. 
indicador, las firmas asistidas del rubro agrícola con origen étnico exhiben un crecimiento mayor. En las conclusiones del documento se realiza una descripción más detallada de al menos tres estudios de caso que representan explícitamente las relaciones, similitudes y mercados comunes que fundamentan el crecimiento y las mejoras en el desempeño empresarial.

\section{Análisis costo-beneficio}

El análisis costo-beneficio es una de las muchas herramientas que ayudan a entender si los programas públicos tienen los efectos esperados. Cuando las intervenciones se realizan con rigurosidad y eficiencia, la herramienta proporciona evidencia sobre las métricas de desempeño, comparando el diferencial resultante entre los costos involucrados y los beneficios que son generados por el programa que se está evaluando. Según las estimaciones realizadas, la relación costo-beneficio del programa es positiva, considerando que los incrementos en ventas ocurridos en el período 2015-2019 sugieren que las empresas asistidas generaron $\$ 2,8$ pesos por cada $\$ 1$ peso invertido (Tabla 5 ).

\section{Conclusiones}

En este artículo hemos examinado los problemas de gestión y la falta de profesionalización que enfrentan las micro y pequeñas empresas para crecer y desarrollarse, y la posibilidad de contrarrestarlos de manera efectiva a través de la provisión de servicios empresariales.

Aunque resulta difícil establecer de forma precisa las razones "últimas" que subyacen bajo estas mejoras empresariales, a raíz de la amplia diversidad de emprendimientos y negocios asistidos por el programa, parece razonable concluir que revelan una profunda transformación en las empresas asesoradas, lo que condice con los impactos originalmente previstos por Sercotec.

Esta forma de causalidad aparece al analizar las encuestas de satisfacción e impacto económico, donde fue posible evidenciar que los resultados obtenidos a nivel cuantitativo y cualitativo son fruto de una serie de acciones encaminadas a resolver deficiencias internas y problemáticas comunes, entre ellas, superar los problemas de acceso a nuevos mercados, acceder a financiamiento y gestionar de forma más eficiente los recursos y capacidades de la empresa.

Las áreas de asistencia examinadas (administración de empresas, desarrollo y consolidación de modelos y planes de negocios, marketing y ventas y acceso a financiamiento) respondieron a la lógica que subyace al diagnóstico de intervención inicial que enfrentó cada segmento asistido. En tal sentido, las diversas medidas implementadas en cada empresa explican algunos de los cambios en las rutinas y conductas "gerenciales", entre otras

Tabla 5. Análisis costo-beneficio (2015-2019).

\begin{tabular}{|c|c|c|c|c|c|}
\hline & Año 1 & Año 2 & Año 3 & Año 4 & \\
\hline & $\overline{2015-2016}$ & $2016-2017$ & $2017-2018$ & $2018-2019$ & Acumulado \\
\hline SERCOTEC & 197.921 .000 & 203.990 .000 & 205.510 .000 & 205.510 .000 & 812.931 .000 \\
\hline Universidad de Tarapacá & 216.900 .000 & 108.180 .000 & 108.180 .000 & 108.180 .000 & 541.440 .000 \\
\hline Otros aportes & 16.000 .000 & 16.000 .000 & 19.000 .000 & 19.000 .000 & 70.000 .000 \\
\hline Costo Operacional & 430.821 .000 & 328.170 .000 & 332.690 .000 & 332.690 .000 & 1.424 .371 .000 \\
\hline Aumento en ventas & 205.445 .320 & 1.050 .391 .874 & 1.552 .453 .210 & 1.271 .362 .396 & 4.079 .652 .800 \\
\hline $\begin{array}{l}\text { relación costo-beneficio medido por el aumento } \\
\text { en ventas }\end{array}$ & $-0,5$ & 3,2 & 4,7 & 3,8 & 2,8 \\
\hline Recaudación Fiscal (IVA) & 88.474 .351 & 459.981 .300 & 1.176 .881 .476 & 651.533 .036 & 2.376 .870 .164 \\
\hline $\begin{array}{l}\text { Relación Costo-Beneficio medido por el aumento } \\
\text { en recaudación fiscal }\end{array}$ & $-0,2$ & 1,4 & 3,5 & 2,0 & 1,7 \\
\hline
\end{tabular}

Fuente: Elaboración propia a partir de Neoserra. 
dimensiones internas, que fueron abordadas a lo largo de cada una de las intervenciones.

Considerando las variables de corte longitudinal, las diferencias empresariales existentes en función del género, tamaño, sector económico y origen étnico explican muchos de los comportamientos observados en los clientes asistidos. Aunque las trayectorias y experiencias empresariales son muy heterogéneas, las principales tendencias indican que las empresas lideradas por mujeres y empresas gacelas $(\mathrm{N} 3)$ han tenido un mejor desempeño en la tasa de aumento en ventas.

En cuanto a la magnitud de los impactos generales, destaca el crecimiento empresarial de los propietarios de origen étnico. Este hallazgo entra dentro de lo esperable, por tratarse, en primer término, de una región con una fuerte presencia de población indígena, y en segunda instancia, por la notoria transformación y cambios de actitud de microempresarias en los rubros de servicios, agricultura y manufactura artesanal (artesanía aymara con identidad local). En este último caso, considerando que las teorías evolutivas tienen una base empírica de gran valor explicativo, los patrones históricos del pueblo aymara determinan también sus comportamientos y singularidades comerciales.

Por lo tanto, se debe tener en cuenta que los procesos de transferencia de conocimiento en este segmento de clientes condicionan favorablemente sus prácticas de gestión y desempeño económico.

Los mejores desempeños empresariales identificados, especialmente del sector agrícola, exhiben ciertas analogías en la mayoría de las áreas de asistencia empresarial abordadas en el largo plazo con apoyo del Centro de Negocios.

En este contexto, las empresas agrícolas con mejor desempeño económico participan simultáneamente en un proyecto colectivo denominado Pampa Concordia. Las firmas son relativamente jóvenes y se caracterizan por cultivar y comercializar hortalizas de especialidad con innovadores sistemas productivos bajo cubierta (tomate cherry, albahaca y capulí). Estas empresas se destacan por una sostenida capacidad de identificar nuevas oportunidades de mercado y hacer un uso eficiente de sus recursos productivos. Esta última situación es clave, ya que las condiciones de suelo y clima local las inducen a innovar permanentemente, utilizando tecnologías productivas de alta precisión procedentes de Israel y España. Destacan en este análisis empresas agrícolas gacelas o de nivel 3.
$\mathrm{Al}$ analizar los planes de asistencia empresarial, las empresas presentan ciertas similitudes. Entre ellas, los propietarios son de origen aymara, profesionales universitarios y tienen un importante historial asociativo. La principal motivación para el uso de la asesoría incluyó el desarrollo de planes y modelos de negocios, identificación de nuevos mercados, apoyo especializado para la obtención de certificaciones (BPA, Global Gap) y asistencia para la búsqueda de financiamiento. Es importante mencionar que las empresas asistidas comercializan sus productos en mercados más rentables, lo cual explica su mejor desempeño empresarial.

Otro elemento que caracteriza a las empresas señaladas es su continua capacidad de experimentación y transmisión de información, fomentando relaciones horizontales entre las mismas empresas, clientes y proveedores de insumos y tecnologías agrícolas. En este contexto, el Centro de Desarrollo de Negocios proporciona una serie de conexiones comerciales, asesorías especializadas y oportunidades de formación, influyendo positivamente en el trabajo en red.

Por otra parte, la obtención de financiamiento con apoyo del programa responde también a la reputación empresarial de las firmas y su inherente capacidad de presentar proyectos más innovadores y competitivos. Las iniciativas agrícolas con apoyo del centro responden muy bien a la tasa de colocación de financiamiento obtenido, incluyendo proyectos DFL15 de CORFO, Proyectos Multisectoriales de SERCOTEC, obtención de financiamiento bancario e inversión con capital ahorrado. Indudablemente, el conjunto de empresarios asistidos (450 por año) también hace un uso intensivo de las asesorías y capacitaciones, especialmente para contar con mejores capacidades para atraer fondos y explotar sus ventajas competitivas.

Desde una perspectiva institucional, el análisis general del programa puso de manifiesto que el punto de equilibrio previsto para el Centro de Arica se logró en el tercer año de operación (2017-2018), donde el porcentaje de clientes con aumentos en ventas alcanzó a un $31 \%$. En este mismo sentido, según la clasificación de clientes, el $10,4 \%$ de los emprendimientos asistidos (N1) muestran incrementos en ventas, mientras que el $35,3 \%$ de la cartera de empresas establecidas (N2) y el $80 \%$ de las empresas gacelas (N3) atribuyen al programa sus aumentos en ventas. 
Respecto del análisis costo-beneficio, se concluye que los incrementos en ventas durante el período 2015-2019, como resultado de las mejoras en el rendimiento de los clientes asistidos, generaron $\$ 2,8$ pesos por cada $\$ 1$ peso invertido. Por tal motivo, la evidencia presentada en este documento indica que los clientes asesorados generan más oportunidades de ventas, así como empleos y obtención de financiamiento, creando positivos retornos fiscales y justificando su continuidad en el tiempo.

\section{Agradecimientos}

Se agradecen especialmente los comentarios y sugerencias realizadas por el doctor Jorge Katz, académico del Departamento de Economía e investigador del Centro INTELIS de la Universidad de Chile.

Cabe mencionar que este artículo ha sido desarrollado en el marco del Programa de Doctorado en Ciencias Jurídicas y Económicas por la Universidad Camilo José Cela - España.

\section{Literatura citada}

America's Small Business Development Centers. Economic Impact. Disponible en: America's Small Business Development Centers (SBDC). Disponible en: https://americassbdc.org/ about-us/a-brief-history/ Consultado: 31/jul/2019

Bloom, N.; Van Reenen, J.

2007. Measuring and explaining management practices across firmas. The Quarterly Journal of Economics, 122: 1351-1408.

Centro de Sistemas Públicos de la Universidad de Chile. 2017. Informe de evaluación de impacto de los Centros de Desarrollo de Negocios en Chile. En: Centro de Sistemas Públicos de la Universidad de Chile. Santiago, Chile. pp. 15, 2017.

Eurostat-OECD.

2007. Manual on Business Demography Statistics. European Commission. Luxemburgo. pp. 3-99.
Ministerio de Economía, Fomento y Turismo.

2014. Agenda de productividad, innovación y crecimiento (2014-2018). En: Ministerio de Economía, Fomento y Turismo. Santiago, Chile. pp. 60-63.

Nelson, R.; Winter, S.

1982. An Evolutionary Theory of Economic Change. Cambridge University Press. Massachusetts and London, England. pp. 3-371.

Penrose, E.

1959. The Theory of the Growth of the Firm. Oxford University Press. England. pp. 1-30

SERCOTEC.

2018. Los Centros de Desarrollo de Negocios en Chile. Sercotec. Santiago, Chile. pp. 2-30.

Tarziján, J.

2009. Fundamentos de Estrategia Empresarial. Quinta Edición. Ediciones UC. Santiago, Chile. pp. 79-99. 